\title{
LES REPRÉSENTATIONS SOCIALES DES FUTURS ENSEIGNANTS DU QUÉBEC SUR LE RÔLE DE L’APPRENTISSAGE MOBILE COMME ÉTUDIANTS'1
}

\author{
PONTES, Renata Lopes Jaguaribe (Canadá, Québec) $)^{1 *}$; \\ KARSENTI, Thierry (Canadá, Québec) ${ }^{1 *}$ \\ ${ }^{1}$ Université de Montréal \\ ORCID ID: https://orcid.org/0000-0003-2347-2302* \\ ORCID ID: https://orcid.org/0000-0003-1733-4608*
}

\begin{abstract}
RÉSUMÉ
L'apprentissage mobile se déroule à travers des interactions sociales et des contenus par l'usage de dispositifs électroniques et personnels. Pour L'unesco, il représente une des solutions pour contribuer à une formation des enseignants capable de répondre aux besoins éducatifs du XXle siècle. Dans le contexte québécois, la formation initiale des enseignants met en évidence l'importance de l'usage des TICs de façon à soutenir leur apprentissage. À partir de la théorie des représentations sociales, cette recherche vise à connaitre les représentations des futurs enseignants québécois sur le rôle de l'apprentissage mobile pour comprendre comment cet apprentissage peut soutenir leurs études. Une méthodologie qualitative a été employée, dont 18 entrevues avec étudiants de baccalauréat en enseignement. Les résultats montrent que l'apprentissage mobile est déjà une réalité dans la vie de ces étudiantes, dont la fonction est de servir à compléter les enseignements reçus dans les cours à l'université.
\end{abstract}

\section{MOTS-CLÉS}

Apprentissage mobile. Futurs enseignants. Représentations sociales.

\section{AS REPRESENTAÇÕES SOCIAIS DOS FUTUROS PROFESSORES DO QUÉBEC SOBRE O PAPEL DA APRENDIZAGEM MÓVEL COMO ALUNOS}

\begin{abstract}
RESUMO
A aprendizagem móvel ocorre por meio de interações sociais e de conteúdo pelo uso de dispositivos eletrônicos e pessoais. Para a Organização das Nações Unidas para a Educação, a Ciência e a Cultura, representa uma das soluções para contribuir com uma formação de professores capaz de atender às necessidades educacionais do século XXI. No contexto do Québec, a formação inicial de professores destaca a importância do uso das tecnologias da informação e comunicação para apoiar sua aprendizagem. A partir da teoria das representações sociais, esta pesquisa tem como objetivo conhecer as representações dos futuros professores do Québec sobre o papel da aprendizagem móvel para entender como essa aprendizagem pode auxiliar seus estudos. A metodologia qualitativa foi utilizada por meio de 18 entrevistas com estudantes de bacharelados em Educação. Os resultados mostram que a aprendizagem móvel já é uma realidade na vida dos estudantes. Ela é, no entanto, um tipo de aprendizagem principalmente utilizado para complementar os conhecimentos vistos na universidade.
\end{abstract}

\section{PALAVRAS-CHAVE}

Aprendizagem móvel. Futuros professores. Representações sociais.

1 O presente trabalho foi realizado com apoio da Coordenação de Aperfeiçoamento de Pessoal de Nível Superior - Brasil (Capes) - Código de financiamento 001.

Educação \& Formação, Fortaleza, v. 4, n. 11, p. 24-40, maio/ago. 2019

DOI: https://doi.org/10.25053/redufor.v4i11.1179

http://seer.uece.br/redufor 


\title{
THE SOCIAL REPRESENTATIONS OF FUTURE TEACHERS OF QUÉBEC ON THE ROLE OF MOBILE LEARNING AS STUDENTS
}

\begin{abstract}
Mobile learning takes place through social interactions and content by the use of electronic and personal devices. For Unesco, it represents one of the solutions to contribute to teacher training able to meet the educational needs of the 21st century. In the Quebec context, initial teacher training highlights the importance of using ICTs to support their learning. Starting with the theory of social representations, this research aims to know the representations of the future Quebec teachers on the role of mobile learning to understand how this learning can support their studies. A qualitative methodology was used by 18 interviews with students of Bachelor of Education courses. The results show that mobile learning is already a reality in the lives of female students, however, is a type of learning that would rather be used to complete their academic studies.
\end{abstract}

\section{KEYWORDS}

Mobile learning. Future teachers. Social representations.

\section{INTRODUCTION}

La prolifération des appareils mobiles dans la société d'aujourd'hui est évidente, comme indiqué par Traxler (2011, p. 4): “[...] presque tout le monde possède un [dispositif mobile], même souvent plus d'un. Non seulement ils les possèdent, mais ils investissent aussi beaucoup de temps, d'efforts et de ressources financières pour les choisir, les acheter, les personnaliser et les exploiter".

L'usage des technologies mobiles pour l'apprentissage, aussi connue sous le nom de Mobile Learning (m-learning ou l'apprentissage mobile, en français), offre un éventail de possibilités pour les professionnels de tous les domaines du savoir. En effet, ces derniers cherchent à se mettre à jour au moyen de cours, conférences, vidéos, tutoriels, forums, ainsi qu'au moyen d'autres ressources, disponibles en ligne ou non.

Dans le cas des enseignants des écoles publiques et privées qui font face au défi d'enseigner les digitales natives (PRENSKY, 2001), l'apprentissage mobile permet d'utiliser ces technologies dans le cadre de leur apprentissage professionnel. Ils peuvent également s'approprier de ces technologies dans leur enseignement ainsi que pour soutenir les élèves qui en font déjà usage à l'intérieur et à l'extérieur de l'école.

Notre recherche se concentre sur de futurs enseignants du Québec, province du Canada qui investit de plus en plus dans l'utilisation des technologies mobiles dans

Educação \& Formação, Fortaleza, v. 4, n. 11, p. 24-40, maio/ago. 2019

DOI: https://doi.org/10.25053/redufor.v4i11.1179

http://seer.uece.br/redufor 
l'éducation de base. La législation régissant la formation initiale des enseignants $d u$ Québec désigne l'intégration des TIC comme une compétence que le futur enseignant doit développer.

Pour favoriser le développement de cette compétence, les cours de formation initiale pour les enseignants au Québec proposent, dans leurs programmes, des études portant sur l'utilisation des TIC dans l'enseignement et l'apprentissage.

Les projets déjà mis en œuvre dans certaines écoles renforcent davantage la nécessité de l'utilisation pédagogique de ces technologies par les enseignants dès leur formation initiale. Après tout, ces derniers travailleront peut-être bientôt dans des écoles équipées de tablettes et d'ordinateurs portables.

En outre, les technologies mobiles font partie de la vie quotidienne des gens. En raison du coût moins élevé de cette technologie et grâce à l'amélioration des réseaux numériques, de nombreuses personnes ont recours à des appareils mobiles en premier choix afin de se connecter à Internet (JOHNSON et al., 2011). À la fin de 2014, le nombre d'abonnements à la large bande mobile atteint 2,3 milliards, ce qui signifie $32 \%$ de la population mondiale, soit cinq fois plus qu'en 2008. D'ailleurs, dans les pays développés, la diffusion de la large bande mobile atteint $84 \%$ de la population (ITU, 2014). Au Québec, $80,4 \%$ des personnes de 18 à 44 ans possèdent au moins un téléphone intelligent, une tablette ou un baladeur numérique et $78,1 \%$ des foyers sont branchés à Internet (CEFRIO, 2013).

À partir des éléments énoncés (législation, accès à des cours sur les TIC pendant la formation initiale et projets de technologie mobile dans les écoles), nous remarquons qu'une exigence et un besoin sociaux se font sentir. Nous constatons de plus que les enseignants devraient s'instruire davantage quant à l'usage pédagogique des technologies mobiles, embrassant les pratiques de l'apprentissage mobile dans leur apprentissage et leur enseignement, ce qui soulève les questions suivantes: quelles sont les représentations des étudiants québécois sur l'apprentissage mobile pour apprendre en tant qu'étudiants?

Dans cet article-ci, nous détaillons les définitions et caractéristiques de l'apprentissage mobile, ainsi que la conception de la théorie des représentations sociales, théorie utilisée pour soutenir notre recherche.

Educação \& Formação, Fortaleza, v. 4, n. 11, p. 24-40, maio/ago. 2019 


\subsection{APPRENTISSAGE MOBILE: DÉFINITIONS ET CARACTÉRISTIQUES}

L'utilisation des technologies mobiles dans l'éducation encourage un nouveau type d'apprentissage. Les chercheurs ne parviennent pas encore à un consensus sur la définition de l'apprentissage mobile. Ce phénomène peut être causé par le fait que ce domaine est très récent et que le M-Learning et ses applications sont encore en évolution (PENG et al., 2009; WINTERS, 2006).

Parmi les définitions rencontrées dans la littérature, Ally (2009) souligne que l'apprentissage mobile est rendu possible par l'utilisation de la technologie mobile sans fil, laquelle permet à toute personne l'accès à des informations et à des matériels d'apprentissage. Kinash, Brand et Mathew (2012) définissent plus largement ce type d'apprentissage, le voyant plutôt comme l'usage, dans des contextes éducatifs, de dispositifs portables avec une capacité de connexion à Internet.

Certaines définitions encore plus récentes sur l'apprentissage mobile retirent l'accent mis sur la simple utilisation de la technologie mobile et mettent surtout en évidence la mobilité que ces technologies peuvent offrir aux étudiants et les avantages que ces appareils peuvent ajouter à leur apprentissage. II y va ainsi de la définition de Crompton (2013) dans laquelle l'apprentissage mobile est vu comme un apprentissage qui se déroule dans des contextes multiples, qu'ils soient formels (université, école) ou informels (dans un espace non académique), à travers des interactions sociales et de contenu en utilisant des dispositifs électroniques et personnels.

Les principales caractéristiques de l'apprentissage mobile sont intrinsèquement associées à la connectivité, la commodité, la collaboration et la personnalisation que les appareils mobiles peuvent créer pour l'apprentissage (KUKULSKA-HULME, 2005). Toutefois, parmi ces caractéristiques, la mobilité apparait comme la plus remarquable, y compris dans le terme même "apprentissage mobile".

Cette mise en contexte précise que l'apprentissage mobile a lieu quand l'apprentissage est à la portée de l'apprenant. Néanmoins, l'apprenant n'est pas nécessairement en mouvement. Par conséquent, la pratique de l'apprentissage mobile implique que l'on peut aussi bien être à la maison, dans la salle de classe d'une école ou d'une université qu'être en déplacement dans un autobus.

Educação \& Formação, Fortaleza, v. 4, n. 11, p. 24-40, maio/ago. 2019 
Dans cette recherche, nous envisageons donc l'apprentissage mobile comme un type d'apprentissage qui tire parti des technologies mobiles (représentées par les appareils mobiles pouvant se connecter à Internet) et ses caractéristiques intrinsèques permettant l'accès à des contenus pédagogiques et à des informations en tout moment, en tout endroit, et qui facilitent la communication et la création de contenus dans des contextes éducatifs formels et informels.

\subsection{LA THÉORIE DES REPRÉSENTATIONS SOCIALES}

Le psychologue Serge Moscovici formule la théorie des représentations sociales à partir du concept de représentations collectives de Durkheim et des études de Piaget, Vygotski, Saussure, Lévy-Bruhl et Freud (ALEXANDRE, 2004; ARRUDA, 2002). II y a plusieurs définitions des représentations sociales. Selon Moscovici (1961, p. 27-28), la représentation sociale est:

\footnotetext{
Un corpus organisé de connaissances et une des activités psychiques grâce auxquelles les hommes rendent la réalité physique et sociale intelligible, s'insèrent dans un groupe ou un rapport quotidien d'échange, libèrent les pouvoirs de leur imagination.
}

Jodelet (1989, p. 36), pour sa part, conceptualise la représentation sociale comme "[...] une forme de connaissance, socialement élaborée et partagée, ayant une visée pratique, et concourant à la construction d'une réalité commune à un ensemble social”. Pour Abric (1989, p. 188), une représentation sociale est "[...] déterminée à la fois par le sujet lui-même (son histoire, son vécu), par le système social et idéologique dans lequel il est inséré et par la nature des liens que le sujet entretient avec ce système social". Elle est une construction toujours contextualisée qui peut se manifester sous diverses formes: images, concepts, catégories et théories, par exemple (SPINK, 1993).

Moscovici (1984) explique qu'une représentation sociale peut être construite au moyen de deux types de procédures: l'objectivation et l'ancrage. L'objectivation permet que de nouveaux concepts abstraits deviennent concrets, tangibles, c'est-à-dire qu'ils deviennent une réalité acquise au sein d'un groupe social. Quant à l'ancrage, il permet à son tour l'intégration de ces nouvelles informations au sein des représentations déjà définies. Par conséquent, ces nouvelles informations s'ajoutent aux précédentes afin de 
créer de nouveaux concepts. Ce processus permet de transformer quelque chose de non familier en un objet familier.

La théorie exposée par Moscovici engendre trois approches différentes développées par les théoriciens Denise Jodelet, Willem Doise et Jean-Claude Abric. À partir d'une approche structurale, Abric expose la Théorie du Noyau Central qui se propose d'expliquer les processus de formation et d'organisation des représentations sociales. Pour Abric (1984, p. 18), une représentation est un ensemble d'informations, d'attitudes, de croyances et d'opinions acquises par un individu par rapport à un objet donné et "toutes les représentations sont organisées autour de noyaux". Ce noyau est un élément fondamental puisqu'il organise les éléments de la représentation et leur donne un sens (ABRIC, 1984; FLAMENT, 1989).

C'est au moyen de cette théorie que nous pouvons connaitre au-delà du contenu des représentations sociales l'organisation des contenus de ces représentations à partir de l'identification de ses systèmes. Les représentations sociales sont organisées en deux systèmes: le système périphérique que contiennent des éléments capables d'un changement plus rapide et le système central composé d'éléments plus stables.

D'après Flament (1989), chaque système accomplit des fonctions qui caractérisent la représentation et qui influencent les pratiques et les prises de décisions des individus. Ainsi, connaître le contenu des représentations sociales des enseignants sur l'apprentissage mobile et comment ces représentations sont organisées, c'est-à-dire, quels sont les éléments qui constituent ces contenus, est important pour exploiter les représentations que les enseignants ont des technologies mobiles pour apprendre.

\section{METODOLOGIE}

Notre choix de l'approche qualitative est confirmé par les caractéristiques de la méthodologie qualitative décrites par Bogdan et Biklen (1994), qui sont intrinsèquement liées aux objectifs de cette recherche. Selon ces auteurs, la recherche qualitative est plus intéressée aux processus et aux significations qui se posent pendant ces périodes.

L'objectif de cette recherche justifie l'importance de comprendre le processus par lequel les sujets passent pour pouvoir vérifier comment le problème se manifeste. Nous ne sommes pas intéressées à présenter ou à analyser seulement les représentations

Educação \& Formação, Fortaleza, v. 4, n. 11, p. 24-40, maio/ago. 2019

DOI: https://doi.org/10.25053/redufor.v4i11.1179

http://seer.uece.br/redufor 
des technologies mobiles par les futurs enseignants, mais nous voulons aussi comprendre ces enseignants eux-mêmes, exploiter leurs perceptions sur l'apprentissage mobile et aussi connaitre comment ils utilisent ces technologies.

Selon Bogdan et Biklen (1994), la recherche qualitative est descriptive, puisque les données collectées se présentent sous la forme de mots ou d'images plutôt que de chiffres. Les instruments de collecte des données utilisées dans cette étude - entrevues - sont descriptifs. Ce type de matériau est riche et précis en détail et peut être utilisé pour appuyer les allégations ou clarifier les points de vue (LÜDKE; ANDRÉ, 1986). Visant à interpréter la signification de ce processus dans sa forme la plus authentique, nous utilisons des entrevues semi-dirigées comme méthodes de collecte de données.

\section{1 ÉCHANTILLON/COLLECTE DES DONNÉES}

Notre recherche a été éfectuée avec des étudiants inscrits en troisième et quatrième années du baccalauréat en enseignement au préscolaire, enseignement primaire et enseignement secondaire à l'Université de Montréal, à Montréal, dans la province du Québec, au Canada.

La sélection exclusive d'étudiants finissants (de troisième et quatrième années) est due au fait que ce public a probablement déjà vécu des expériences formelles et informelles avec les technologies mobiles tout au long de leur apprentissage ainsi qu'en milieu scolaire pendant les stages.

Comme notre recherche a comme objectif de mieux connaitre les représentations sociales des futurs enseignants québécois sur l'apprentissage mobile en tant qu'étudiants universitaires; nous avons utilisé l'entrevue, l'une des principales méthodes de collecte de données dans la perspective de la recherche qualitative. Selon Van der Maren (1996, p. 312), l'avantage majeur de l'entretien par rapport aux autres outils est qu'il permet d'obtenir “[...] des informations sur les perceptions, les états affectifs, les jugements, les opinions, les représentations des individus, à partir de leur cadre personnel de référence et par rapport à des situations actuelles".

L'entrevue est une méthode de collecte de données très utilisée dans les recherches sur les représentations sociales (KOUAWO, 2011). Selon Abric (1994),

Educação \& Formação, Fortaleza, v. 4, n. 11, p. 24-40, maio/ago. 2019 
l'entrevue constitue une méthode indispensable à toute étude sur les représentations sociales.

Les entrevues varient en fonction du degré de structuration. À l'occasion de cette recherche, nous avons effectué des entrevues individuelles avec 18 futurs enseignants québécois, c'est-à-dire que nous nous sommes concentrées sur des questions spécifiques ou guidées par des questions générales provenant du cadre théorique de la recherche (BOGDAN; BIKLEN, 1994; KARSENTI; SAVOIE-ZAJC, 2000).

\subsection{ANALYSE DES DONNÉES}

L'analyse des données a été effectuée selon les critères de la stratégie d'analyse de contenu décrit par Van der Maren (1996, p. 406) qui "s'intéresse à l'information contenue dans un message". Selon Paillé (1996), l'analyse de contenu est une méthode qui vise à fournir une compréhension textuelle.

Pour effectuer l'analyse de contenu, nous avons utilisé le logiciel d'analyse qualitative QDA Miner qui, en plus de faciliter la réalisation du codage des matériaux, dispose également de fonctionnalités qui ont permis la réalisation du processus de codage inverse, le contre-codage et l'accord inter-juge.

Tout d'abord, les 18 entretiens ont été transcrits. Avant de commencer le processus de codage, nous avons relu tous les verbatim. Après la relecture, nous avons codé les entrevues avec le logiciel QDA Miner, ce qui nous a permis d'élaborer progressivement une grille de codage. Après avoir codifié l'ensemble des verbatim avec une grille de codage stabilisé, nous sommes passées au processus de contre-codage. Les taux d'accord inter-juges à partir de la réalisation du contre-codage ont été de $85 \%$ pour les entrevues des étudiants.

\section{RÉSULTATS}

Lors des entrevues, les représentations des étudiants québécois concernant le rôle de l'apprentissage mobile pour leurs études se sont révélées et à partir de l'analyse des données nous en sommes venues à une seule catégorie que montre que l'apprentissage mobile est perçu par eux comme un complément pour soutenir leurs 
cours présentiels pendant la formation initiale. Les 18 étudiants québécois interviewés considèrent que le rôle de l'apprentissage mobile dans leur vie étudiante est complémentaire. Ils indiquent que les technologies mobiles et l'Internet sont des outils qui aident leurs apprentissages des contenus vus dans les cours à l'université, mais qu'elles ne sont pas les éléments principaux, comme le soulignent les extraits suivants: "On peut se servir des technologies mobiles pour complémenter (l'apprentissage), mais jamais comme outil principal" (Étudiant 7). "C'est bien, c'est un complément à ce qu'on apprend à l'école"2 (Étudiant 13). "[...] D'avoir l'apprentissage mobile, c'est plus une complémentarité en fait, une plus-value" (Étudiant 5).

Une étudiant dite que même en l'appréciant, l'apprentissage mobile est un plus pour aider dans les études, puisque ce type d'apprentissage ne peut pas substituer les cours présentiels.

J'aime beaucoup l'apprentissage mobile, mais pas pour tout; pour tout ce qu'il y a en plus, tout ce qui est le travail que ça nous donne, si on a des travaux de session à rendre par exemple on peut toujours aller chercher de l'information ailleurs, on le fait déjà, mais il y a une partie du cours que j'aime bien quand on la voit avec les autres. Je n'aimerais pas que tout le cours soit toute seule. (Étudiant 1).

Ce type d'apprentissage est pratique pour aider à accompagner les lectures faites pendant un cours, par exemple.

C'est vraiment une question d'accompagnement, si j'ai oublié d'imprimer quelque chose, ben je vais aller voir là-dessus. [...] Ce n'est vraiment pas ma première source d'apprentissage, ça reste vraiment juste un accompagnement pour moi les technologies mobiles. (Étudiant 9).

Un autre étudiant décrit le rôle complémentaire de l'apprentissage mobile en se référant aux pratiques quotidiennes qu'il effectue avec ces technologies pour aider dans ses lectures des textes des cours.

\footnotetext{
Pour moi, ça (les technologies mobiles) a toujours été un outil de dépannage, ça a toujours été ça pour moi. Admettons qu'il y a une lecture que je n'avais pas faite, ben je vais aller sur l'application, sur Studium, puis je vais aller sur PDF', puis je vais le lire dans l'autobus. Pour moi ça a toujours été un outil de dépannage. (Étudiant 3).
}

2 L'étudiant utilise le mot "école" pour se référer à l'université.

3 Le PDF (Portable Document Format) est un format de fichier inventé par la compagnie Adobe. II est utilisé pour afficher et partager des documents dans n'importe quel logiciel, matériel ou système d'exploitation. Informations trouvées à l'adresse suivante: <https://acrobat.adobe.com/br/pt/whyadobe/about-adobe-pdf>.

Educação \& Formação, Fortaleza, v. 4, n. 11, p. 24-40, maio/ago. 2019

DOI: https://doi.org/10.25053/redufor.v4i11.1179

http://seer.uece.br/redufor 
Dans la situation explicitée, les aspects de la mobilité et portabilité, intrinsèques aux technologies mobiles, sont soulignés à partir de la pratique de la lecture des textes PDF dans l'autobus. Cette nouvelle possibilité de l'apprentissage mobile renforce la contribution qu'elle peut donner aux études de ces futurs enseignants.

Selon les discours des étudiants, cette complémentarité est possible à cause de la variété des types de contenus (vidéos, audios, textes) qui les aident à comprendre les matières vues pendant les cours présentiels par l'entremise d'un apprentissage plus informel et autonome.

Je trouve que ça peut m'aider de différentes façons à comprendre la matière. Il y a des vidéos, des textes plus audios, ben ça permet d'apprendre d'une autre façon, c'est stimulant puis ça permet de plus nous débrouiller par nous-mêmes. [...] Ben, premièrement si je ne comprends pas une notion, avant d'aller voir le professeur, j'essaie d'aller sur Internet, avoir des renseignements supplémentaires qui pourraient m'aider à comprendre la matière. (Étudiant 13).

Certaines étudiantes ont remarqué que la diversité du matériel de consultation leur permet non seulement de mieux comprendre les matières, mais d'approfondir les contenus, ne se limitant pas seulement aux références données dans le cours et à l'exposé du professeur. En voici des exemples: "Par la masse d'informations, on a plus accès à d'autres informations; si je m'étais limité aux cours que j'ai à l'université, il y a plein de choses que je ne saurais pas, c'est du plus" (Étudiant 1).

Pour une étudiante du cours de Baccalauréat en enseignement de l'Éthique et de la culture religieuse au secondaire, l'apprentissage mobile se passe principalement par l'écriture des synthèses des contenus des cours et de l'usage des applications mobiles disponibles pour approfondir leurs connaissances sur la bible ou autres livres sacrés.

Souvent l'apprentissage mobile ça va être beaucoup des résumés, ça va être des synthèses, souvent il y a des applications qui vont me dire: maintenant que tu as lu la bible, ben voici tu peux comprendre telle chose avec telle application, par exemple, qu'est-ce que l'évangile veut dire, avec une application. Là, on va m'expliquer et je vais pouvoir aller rebondir là-dessus en fait, donc c'est beaucoup pour de la lecture, de l'approfondissement de la compréhension. (Étudiant 5).

Cette même étudiante remarque également que ce type d'apprentissage permet d'avoir "beaucoup plus de choses sous la main". Parmi elles, l'accès rapide à une Bible, une Torah, un Coran qui sont des livres lourds et qui prennent beaucoup d'espace, mas

Educação \& Formação, Fortaleza, v. 4, n. 11, p. 24-40, maio/ago. 2019

DOI: https://doi.org/10.25053/redufor.v4i11.1179

http://seer.uece.br/redufor 
qu'elle peut avoir "au bout du doigt" sur le téléphone cellulaire ou la tablette quand elle les veut pour étudier.

Une autre possibilité d'améliorer l'apprentissage en utilisant ces technologies, qui a été mentionnée par quelques étudiants, est la visualisation des vidéos sur YouTube pour comprendre un contenu spécifique, comme le décrit un étudiant du cours de Baccalauréat en enseignement de l'Éducation physique et à la santé. II pratique l'apprentissage mobile en regardant des vidéos sur YouTube en vue d'apprendre des techniques de mouvement.

C'est sûr pour augmenter mon apprentissage. Oui souvent je vais aller consulter des vidéos surtout dans le domaine de l'éducation physique; souvent on peut apprendre des techniques de mouvement, on va aller souvent visionner une vidéo de professionnels qui prennent le temps de bien démontrer un mouvement. Mais outre cela, oui, souvent je vais utiliser les technologies pour aller chercher de l'information, des articles scientifiques. Oui, je les utilise pour augmenter mes connaissances. (Étudiant 8).

Cette possibilité d'aller chercher des matériaux sur YouTube pour mieux comprendre les contenus du cours est soulignée en d'autres situations. Une étudiante du cours de Baccalauréat en Éducation préscolaire et enseignement primaire dit que son ordinateur portable et son téléphone cellulaire ont été des outils importants pendant son parcours à l'université, principalement pour comprendre des concepts du cours de Mathématique.

\begin{abstract}
Personnellement, j'utilise les technologies tout le temps. Donc dès que je ne comprends pas quelque chose, je vais aller voir sur Internet, sur mon téléphone; je n'ai pas de tablettes, donc l'ordinateur et le téléphone sont vraiment mes outils principaux. J'ai à la maison des ouvrages de référence, mais je ne les utilise plus, je vais directement voir sur Internet; faudrait pas que je te le dise (rire), mais je vais te le dire pareil, donc mon premier cours de mathématiques, j'ai tout appris sur Internet donc je n'ai rien retenu du cours en tant que tel, donc j'allais voir sur Internet tout ce qui transformait des bases..., donc tout se trouve sur Internet, sur You Tube c'est incroyable, je trouve tout là-dedans. (Étudiant 17).
\end{abstract}

L'étudiante ajoute que la possibilité de chercher et d'avoir des informations par vidéos ou textes répondant à ses doutes lui donne beaucoup de liberté et plus de temps pour essayer de suivre ou de comprendre un contenu, puisqu'elle peut relire les textes et voir les vidéos autant de fois qu'elle le souhaite. À son avis, l'apprentissage mobile est complémentaire à l'apprentissage formel puisqu'elle "s'adapte à nous". En plus, elle dit qu'elle évite de poser des questions en classe pour être gênée et avoir peur d'être la

Educação \& Formação, Fortaleza, v. 4, n. 11, p. 24-40, maio/ago. 2019

DOI: https://doi.org/10.25053/redufor.v4i11.1179

http://seer.uece.br/redufor 
seule qui n'ait pas compris ce que le professeur a expliqué. Avec ces technologies elle ne se sent pas “jugée” parce qu'elle n'a pas compris.

En un autre exemple, un étudiant mentionne que les technologies mobiles, dans son cas le téléphone cellulaire et l'Internet, sont des outils qui peuvent aider à la compréhension du contenu en classe pendant un cours, comme il l'explique:

\begin{abstract}
[...] dans un cours s'il y a quelque chose qu'on n'a pas compris, un mot que le professeur dit, parce que parfois les professeurs sont très [...], surtout à l'université, les professeurs sont très ancrés dans leur discipline et ne sont pas de très bons pédagogues non plus, je vous dirais que la plupart des professeurs de l'université ne sont pas formés pour être des enseignants. Ils sont formés pour la recherche, puis ça parait, parfois on dirait qu'ils partent puis quand les cours sont très magistraux, des fois ils partent sur des idées ou des concepts, ça peut être compliqué à comprendre. D'avoir accès au téléphone et d'avoir aussi accès à Internet ben ça permet d'aller voir, de vérifier des choses, comprendre plus rapidement quelque chose qu'on n'a pas nécessairement compris du premier coup. (Étudiant 6).
\end{abstract}

En plus de la praticité de clarifier des concepts et des doutes hors et en classe, un étudiant souligne l'importance de la mobilité des technologies mobiles et de l'accès répandu à Internet pour le rôle complémentaire de l'apprentissage mobile. Pour lui, ces aspects ont élargi les temps et les espaces d'apprentissage au-delà des murs de l'université.

\begin{abstract}
Si on veut aller plus loin, c'est plus facile aussi, parce que les professeurs nous donnent des pistes; souvent on a beaucoup de lectures, on doit lire de telle à telle page, mais moi personnellement ce n'est pas ma passion la lecture, dans les livres en tout cas, mais quand on peut regarder des travaux d'autres personnes sur Internet qui sont en lien avec ça ou même qui ont une opinion différente du professeur, ça peut être intéressant aussi parce que ça élargit nos horizons. En fait on va plus loin dans la matière, mais ce n'est pas lié à l'endroit, on peut faire ça où on veut; je me suis plus approfondi par le fait que I'Internet nous permet de faire ça, mais en fait ça nous permet de faire ça, mais partout, et quand on veut. L'apprentissage mobile nous permet vraiment de faire ces approfondissements personnels quand on veut et où on veut. (Étudiant 11).
\end{abstract}

Dans cet exemple, l'autonomie de pouvoir accéder à des travaux et à des opinions diverses sur un sujet étudié en classe sans avoir une limitation d'horaire et d'endroit est considérée comme une valeur ajoutée que proportionne ce type de technologie. Un autre étudiant met également en évidence cet avantage d'approfondir les lectures à partir de recherches faites sur Internet; par contre, il souligne que si cet usage a un côté pratique, il a simultanément un côté qui peut distraire.

Educação \& Formação, Fortaleza, v. 4, n. 11, p. 24-40, maio/ago. 2019 DOI: https://doi.org/10.25053/redufor.v4i11.1179 
[...] s'il y a quelque chose que je lis puis j'aimerai approfondir, ben je vais sur Internet. J'approfondis comme ça, je passe sur des recherches Wikipédia ou, peu importe, puis le temps que je reviens, je perds le fil tout le temps, alors que si j'avais un livre, j'aurais un fil conducteur dans mes lectures puis je ferai mes recherches après. Je me distrais beaucoup puis finalement dans ma journée en tant que telle dans ma lecture que j'avais à faire, je n'ai presque rien fait tandis que si j'avais un livre, ben j'aurai avancé beaucoup plus. (Étudiant 15).

Réaffirmant la perception du potentiel distrayant des technologies mobiles, un autre étudiant affirme même qu'il ne les utilise seulement que pour des activités nécessaires pour son apprentissage comme écrire un texte, avoir accès à des documents et faires des recherches.

\begin{abstract}
Pour mon apprentissage, je dirais que (les technologies mobiles) c'est assez distrayant (rire). J'essaie de l'utiliser le moins possible. En fait, quand c'est nécessaire pour écrire un texte sur un ordinateur, je l'utilise, mais sinon j'essaie de ne pas lire de texte sur l'ordinateur parce que j'enregistre beaucoup moins quand c'est à l'ordinateur que quand c'est à l'écrit. [...] c'est sûr qu'on en a besoin pour accéder à tous les documents, Internet. Google est un grand ami (rire). En fait, oui, c'est utile pour chercher des choses; ce sont de bons outils de recherche. (Étudiant 10).
\end{abstract}

La distraction est le défi le plus souligné dans les discours des étudiants, comme étant un élément qui rend difficile la pratique de l'apprentissage mobile et qui minimise les bénéfices que ce type d'apprentissage peut apporter à leurs études.

En définitive, pour les futurs enseignants québécois, le rôle de l'apprentissage mobile est vu comme complément à leurs études aux cours présentiels. Ils considèrent les technologies mobiles et Internet comme des outils qui les aident à accéder, partager et produire des contenus et des informations ainsi qu'à mieux comprendre les contenus et à approfondir leur apprentissage.

\title{
4 DISCUSSION/CONCLUSIONS
}

À partir de l'analyse des résultats, nous pouvons conclure que l'apprentissage mobile occupe déjà une place dans la vie étudiante des futurs enseignants québécois. Mais c'est en connaissant les représentations qu'ils se font de ce type d'apprentissage qu'il est possible de mieux comprendre le rôle que les étudiants attribuent à cet apprentissage, puisque les processus de représentation constituent des connaissances du sens commun qui sont élaborées et partagées socialement (DOISE, 1985).

Educação \& Formação, Fortaleza, v. 4, n. 11, p. 24-40, maio/ago. 2019

DOI: https://doi.org/10.25053/redufor.v4i11.1179

http://seer.uece.br/redufor 
À la lumière de la Théorie du Noyau Central et à partir de notre analyse des résultats, nous pouvons affirmer que le rôle de l'apprentissage mobile comme complément pour soutenir l'apprentissage formel constitue le noyau central des représentations sociales des futurs enseignants québécois.

Les représentations sociales sont construites au moyen de deux types de procédures: I'ancrage et l'objectivation, comme nous l'avons vu précédemment. C'est à partir de ces processus que le sujet transforme quelque chose de non familier en un objet familier (MOSCOVICl, 1984).

Les représentations sociales des étudiants québécois sur le rôle de l'apprentissage mobile montrent qu'il est perçu comme un appui pour leur apprentissage formel à l'université. Pour ces étudiants, l'apprentissage mobile est catégorisé comme un complément pour leurs cours présentiels pendant la formation initiale.

Dans les entrevues, les étudiants classifient le rôle de l'apprentissage mobile en utilisant les mots "compléter", "complémenter", "bonus", "secondaire", "outil de dépannage" et "outils supplémentaires". C'est dans le processus d'ancrage que les étudiants transforment leurs perceptions de l'apprentissage mobile en quelque chose de familier à partir des leurs expériences et mémoires.

Au moment que ces mots font place à une image, gagnant une forme concrète, les étudiants à partir du processus d'objectivation associent la perception que l'apprentissage mobile est un complément pour soutenir leurs apprentissages à des pratiques avec des technologies mobiles, à savoir, à l'usage des applications, regarder des vidéos sur YouTube, faire des recherches dans le Google et Wikipédia et se communiquer par Facebook.

Les étudiants québécois pratiquent l'apprentissage mobile surtout pour étudier dans et dehors de la salle de cours. Même s'ils s'en servent davantage à la maison et à l'université, ils se montrent contents de pouvoir les utiliser pendant leurs déplacements, dans l'autobus ou le métro, ou dans les cafés.

Ils voient également ces technologies comme des outils d'appui pour approfondir leur apprentissage à partir de lectures complémentaires et visualisations de vidéos. Une étudiante donne comme exemple l'utilisation du YouTube pour mieux suivre une discipline de mathématique reconnue pour être difficile. Un autre soutien pourvu par ces

Educação \& Formação, Fortaleza, v. 4, n. 11, p. 24-40, maio/ago. 2019 
outils est lié à la communication entre les étudiants pour clarifier des doutes et partager des informations.

Les étudiants rapportent que leurs professeurs n'ont pas l'habitude d'utiliser le Facebook ou WhatsApp, puisqu'ils utilisent plutôt la plateforme d'apprentissage de l'université et le courriel électronique, mais ils utilisent le Facebook Messenger pour se communiquent entre eux. Les québécois sont également connectés principalement au Facebook et YouTube. Selon les données du CEFRIO (2015), celles-ci sont deux plateformes sociales (Facebook avec 62,4 \% et YouTube avec 57,4\%) utilisées par plus de la moitié de la population adulte du Québec.

Les étudiants québécois affirment que l'apprentissage mobile ne peut pas substituer les cours présentiels, puisque, pour eux, l'interaction face à face est très importante. La littérature scientifique sur l'apprentissage mobile soulignait déjà la tendance de ce type d'apprentissage de ne pas substituer l'apprentissage présentiel, mais de servir comme "[...] moyen de compléter le processus d'apprentissage grâce à des appareils mobiles" (UNESCO, 2012).

Nous constatons, toutefois, que plus le cours est difficile, plus les étudiants pratiquent l'apprentissage mobile. C'est le cas des cours liés à la mathématique. Quelques étudiants affirment même que sans les vidéos sur YouTube pour expliquer les contenus de mathématique il serait difficile de comprendre les contenus vus en classe. En même temps qu'il est positif de voir qu'il est utile de pouvoir compter sur des vidéos comme recours complémentaires, cette réalité est préoccupante puisqu'elle signifie que quelque chose ne va pas très bien dans les cours présentiels, qui ne sont pas suffisants pour que les étudiants apprennent.

\section{RÉFÉRENCES}

ABRIC, J.-C. A theoretical and experimental approach to the study of social representations in a situation of interaction. In: FARR, R. M.; MOSCOVICI, S. Social representations. Cambridge: Cambridge University, 1984. p. 169-183.

ABRIC, J.-C. L'étude expérimentale des représentations sociales. In: JODELET, D. Les représentations sociales. Paris: PUF, 1989. p. 205-223.

ABRIC, J.-C. Pratiques sociales et représentations. Paris: Universitaires de France, 1994. 
ALEXANDRE, M. Representação Social: uma genealogia do conceito. Revista Comum, Rio de Janeiro, v. 10, n. 23, p. 122-138, 2004.

ALLY, M. (Ed.). Mobile learning: transforming the delivery of education and training. Athabasca: Athabasca University, 2009.

ARRUDA, A. Teoria das representações sociais e teorias de gênero. Cadernos de Pesquisa, São Paulo. n. 117, p. 127-147, 2002.

BOGDAN, R.; BIKLEN, S. Investigação qualitativa em educação: uma introdução à teoria e aos métodos. Porto: Porto, 1994.

CEFRIO. Netendaces 2015 - Usage du Web, médias sociaux et mobilité. Montreál, 2015. Repéré à : <http://www.cefrio.qc.ca/projets-recherches-enquetes/internet-mediassociauxmobilite/netendances-2015/>.

CEFRIO. Netendances. Montréal, 2013. Repéré à : <http://www.cefrio.qc.ca/ projetsrecherches-enquetes/numerique-generation/netendances-2013/>.

CROMPTON, H. A historical overview of mobile learning: toward learner-centered education. In: BERGE, Z. L.; MUILENBURG, L. Y. (Ed.). Handbook of mobile learning. Florence: Routledge, 2003. p. 3-14.

DOISE, W. Les représentations sociales: définition d'un concept. Paris: Connexions, 1985.

FLAMENT, C. Structure et dynamique des représentations sociales. In: JODELET, D. Les représentations sociales. Paris: PUF, 1989. p. 224-239.

ITU. The world in 2014: ICT facts and 230 figures. Geneva: ITU, 2014.

JOHNSON, L. R. et al. The 2011 horizon report. Austin: The New Media Consortium, 2011.

KARSENTI, T.; SAVOIE-ZAJC, L. Introduction à la recherche en éducation. Sherbrooke: CRP, 2000.

KINASH, S.; BRAND, J.; MATHEW, T. Challenging mobile learning discourse through research: student perceptions of blackboard mobile learn and iPads. Australasian Journal of Educational Technology, v. 28, n. 4, p. 639-655, 2012.

KOUAWO, A. Que pensent les enseignants et les élèves du secondaire des TIC? Une étude des représentations sociales au Niger. 2011. $192 \mathrm{f}$. Thèse (Doctorat en Sciences de l'Éducation - Technopédagogie) - Faculté de Sciences de l'Éducation, Université de Montréal, Montréal, 2001.

KUKULSKA-HULME, A. Mobile usability and user experience. In: KUKULSKA-HULME, A.; TRAXLER, J. Mobile learning: A handbook for educators and trainers. London: Routledge, 2005, p. 45-56. 
LÜDKE, M.; ANDRÉ, M. E. D. A. Pesquisa em educação: abordagens qualitativas. São Paulo: Pedagógica e Universitária, 1986.

MOSCOVICI, S. La psychanalyse: son image et son public. Paris: PUF, 1961.

MOSCOVICI, S. The phenomenon of social representations. In: FARR, R.; MOSCOVICI, S. (Dir.). Social representations. Cambridge: Cambridge University, 1984. p. 3-69.

PAILLÉ, P. De l'analyse qualitative en général et de l'analyse thématique en particulier. Recherches Qualitatives, v. 15, p. 179-184, 1996.

PENG, $\mathrm{H}$. et al. Ubiquitous knowledge construction: mobile learning re-defined and a conceptual framework. Innovations in Education and Teaching International, v. 46, n. 2, p. $171-183,2009$.

PRENSKY, M. Digital natives digital immigrants. On the horizon, v. 9, n. 5, p. 1-6, 2001.

SPINK, M. J. P. O conceito de representação social na abordagem psicossocial. Cadernos de Saúde Pública, Rio de Janeiro, v. 9, n. 3, p. 300-308, 1993.

TRAXLER, J. Introduction. In: TRAXLER, J.; WISHART, J. Making mobile learning work: case studies of practice. Bristol: Centre for Education University of Bristol, 2011.

UNESCO. L'apprentissage mobile pour les enseignants. Thèmes généraux, 2012. Repéré à: <http://unesdoc.unesco.org/images/0021/002164/216452f.pdf>.

VAN DER MAREN, J.-M. Méthodes de recherche pour l'éducation. 2. ed. Montréal: PUM; Bruxelles: De Boeck Université, 1996.

WINTERS, N. What is mobile learning? In: SHARPLES, M. Big issues in mobile learning: Report of a workshop by the Kaleidoscope Network of Excellence Mobile Learning Initiative. Nottingham: University of Nottingham, 2006.

\footnotetext{
Renata Lopes Jaguaribe Pontes (Canadá, Québec) - Université de Montréal

Doutora em Ciências da Educação pela Université de Montréal.

Lattes: <lattes.cnpq.br/8128009877760964>.

E-mails: <renata.lopes.jaguaribe.pontes@umontreal.ca> e <renatajaguar@gmail.com>.
}

\section{Thierry Karsenti (Canadá, Québec) - Université de Montréal}

Doutor em Educação pela Université du Québec à Montréal. Professor da Université de Montréal.

E-mail: <thierry.karsenti@umontreal.ca>.

Recebido em 30 de março de 2019.

Aceito em 12 de abril de 2019.

Educação \& Formação, Fortaleza, v. 4, n. 11, p. 24-40, maio/ago. 2019

DOI: https://doi.org/10.25053/redufor.v4i11.1179

http://seer.uece.br/redufor 